\section{Kinder und Jugendliche in suizidalen Krisen}

Verena Leutgeb, Elise Steiner, Elisabeth Waibel-Krammer. Kinder und Jugendliche in suizidalen Krisen. Wie Eltern helfen können ein Ratgeber. Göttingen: Hogrefe Verlag 2019. 77 Seiten, 14,95 Euro, ISBN 978-380-172-965-3

Suizidale Gedanken und suizidale Krisen sind gerade im Jugendalter und jungen Erwachsenenalter nicht selten. Es ist wichtig, das Thema Suizidalität, aber auch häufig zugrunde liegende Depressionen, in der Bevölkerung bekanntzumachen, um Warnzeichen zu erkennen und nötigenfalls Suizid verhindern zu können. Das vorliegende
Buch ist deshalb ein wichtiger Ratgeber für alle Familien mit Kindern und Jugendlichen, in denen es suizidale Gedanken, Anzeichen für Depression und Selbstmordgedanken gibt.

Im Buch werden sehr gut gegliedert Ursachen und Hintergründe zu suizidalen Gedanken, Zeichen und Häufigkeit sowie die Entwicklung von Selbstmordgedanken beschrieben. Es ist gut, dass dabei immer wieder betont wird, dass Suizidalität und Selbstmordgedanken auch bei gesunden Jugendlichen und jungen Erwachsenen gerade in der Pubertät ein Thema sind. Professionelle Hilfsangebote müssen be- kannt sein und niederschwellig zur Verfügung stehen.

Das Buch bietet Hilfestellungen zum direkten Gespräch mit Betroffenen, gibt Notrufnummern, Hinweise für Online-Beratung und Links zu Informationen und Hilfsangeboten sowie schließlich Hinweise zu Selbsthilfegruppen für Eltern, die ein Kind durch Suizid verloren haben. Das Buch wird abgerundet durch Literaturempfehlungen und ist insgesamt handlich und sehr übersichtlich zur Orientierung und schnellen Hilfestellung zu empfehlen.

Wieland Kiess, Leipzig 